signs - insbesondere multifaktorielle - werden ignoriert. Auch hier erfolgt wieder der Versuch, Parallelen zwischen Forschung und journalistischer Praxis zu ziehen, und auch hier scheitert er. Die Beispiele für journalistische „Experimente“ erinnern nicht ohne Grund an Garfinkel'sche Krisenexperimente - eine klassische Methode interpretativer Sozialforschung - und weisen keinen nennenswerten Bezug zu den hier beschriebenen Forschungsstrategien auf.

Als Beispiel für „angewandte Sozialforschung" dient ein Kapitel zur Wahlforschung - angemessen für die Zielgruppe der Journalisten, verfehlt für die der Kommunikationswissenschaftler. Leider beschränken sich die Ausführungen auf eine Aufzählung von gängigen Praktiken angewandter Forschung.

Die abschließenden Betrachtungen zur "Qualität der Berichterstattung über empirische Sozialforschung" sind als Leitfaden für die journalistische Arbeit gedacht und sind als solche mit Einschränkungen brauchbar. Denn hier geht es nicht um Sozialforschung im Allgemeinen, sondern um kommerzielle Umfrageforschung. Dabei werden die üblichen Spannungsfelder problematisiert: zu wenig Informationen über die Umfragen, fragwürdiges Interesse des Publikums an methodischen Details. Die angeführten Beispiele verdeutlichen, wo das Problem tatsächlich liegt: Die angewandte Umfrageforschung kennt genügend Möglichkeiten, trotz Qualitätsstandards Ergebnisse zu veröffentlichen, deren „Objektivität" mehr als zweifelhaft ist, weil die Praxis der medialen Berichterstattung keine adäquate Überprüfung zulässt.

Fazit: Die Nützlichkeit des Buchs als Einführung in die empirische Sozialforschung für Journalisten leidet unter dem wenig überzeugenden Versuch, wissenschaftliche und journalistische Zugänge zur Realität in Beziehung zu setzen.

Wolfgang Eichhorn

\section{Margreth Lünenborg}

\section{Journalismus als kultureller Prozess}

Zur Bedeutung von Journalismus in der Mediengesellschaft - ein Entwurf

Wiesbaden: VS, 2005. - $238 \mathrm{~S}$.

ISBN 3-531-14462-6

Die Klärung der Rolle und Bedeutung von Jour- nalismus in der modernen Mediengesellschaft ist eine Frage, die es mit Blick auf die aktuell stattfindenden Transformationsprozesse immer wieder neu zu beantworten gilt. Eine kulturtheoretische Perspektive kann hierzu ohne Zweifel einen wichtigen Beitrag leisten, da sie der Veränderungsdynamik und steten Neupositionierung von Journalismus Rechnung trägt. Margreth Lünenborg hat sich diesem Projekt bereits seit mehreren Jahren verschrieben - insofern ist ihre Habilitationsschrift die konsequente Fortführung eines langfristig angelegten Forschungsprogramms.

Das Ziel der Arbeit definiert die Autorin mit der Erarbeitung eines theoretischen Konzepts für die Beschreibung der Bedeutung von Journalismus in der Gesellschaft aus kulturtheoretischer Sicht. Bei der Analyse von Journalismus als dem zentralen Ort der kulturellen Selbstverständigung der Mediengesellschaft plädiert Lünenborg für ein integratives Konzept der Journalistik: Die zentrale These lautet, dass sich die gesellschaftliche Bedeutung von Journalismus nur im Zusammenwirken von Medienproduktion, Medientext und Medienrezeption erschließt. Denn Sinn, so die Autorin weiter, wird nicht auf Basis der Kommunikationsabsichten von Journalisten geschaffen, sondern entsteht auf der Grundlage der Bedeutungszuweisungen durch das Publikum. Durch die Betonung der Rezeptionsdimension grenzt Lünenborg ihren Ansatz einer kulturtheoretisch orientierten Journalistik von anderen, eher kommunikatorzentrierten Konzepten ab. Damit wird der 238 Seiten starke Band zweifellos auf das Interesse nicht nur von Journalismusforschern stoßen.

Das Buch ist übersichtlich in insgesamt drei Teile und acht Kapitel gegliedert. Im ersten Kapitel beschäftigt sich Lünenborg kritisch mit der Exklusion des Publikums in der aktuellen Journalismusforschung. Diesen Mangel hat die Autorin auch in früheren Arbeiten problematisiert, etwa in einer Kontroverse, die vor sieben Jahren in dieser Zeitschrift ausgetragen wurde. Konkret bemängelt Lünenborg den einseitigen Fokus auf Kommunikatoren und Inhalte und fordert, die „Arbeitsteilung“ zwischen Journalismusforschung und Publikums- und Wirkungsforschung durch einen integrativen Ansatz zu überwinden. Darüber hinaus attestiert sie der traditionellen Journalismusforschung einen verengten Journalismusbegriff: Journalismus lasse sich nicht auf Formen des (poli- 
tischen) Nachrichtenjournalismus reduzieren. Dieser Vorwurf wirkt zuweilen etwas pauschal, insbesondere dort, wo die Autorin die von ihr kritisierte Perspektive recht einseitig darstellt. Die Erforschung von journalistischen Praktiken jenseits des „klassischen“ Nachrichtenjournalismus (z. B. Service-, Prominenten- und Sportjournalismus) mag zwar quantitativ noch unterbelichtet sein, sie ist aber durchaus im Fach vertreten.

In den zwei folgenden Kapiteln arbeitet Lünenborg auf einen integrativen Ansatz für die Journalistik hin und nimmt hierbei dezidiert eine Perspektive im Nexus der Cultural Studies ein. Das „Cultural-Studies-Projekt" wird als konstruktivistische, kulturalistische, prozessuale, kontextuelle, diskursive und kritische Theorie diskutiert, wobei der Nutzen und die grundsätzliche Perspektiverweiterung für die kulturorientierte Journalismusforschung darin besteht, dass die Bedeutungsproduktion journalistischer Medienangebote im Zirkel bzw. Zusammenwirken von Produktion, Text und Rezeption betrachtet wird.

Besonders wertvoll und hilfreich ist Lünenborgs Versuch, eine umfassende Genretheorie am Beispiel des TV-Journalismus zu entwerfen. Das Kapitel zu den Elementen einer journalistikwissenschaftlichen Genretheorie ist damit insgesamt das Lesenswerteste. Lünenborg löst die Genretheorie dabei von ihrem latenten Essenzialismus und betrachtet Genres als bedeutungsgenerierende Strukturen, die im Wechselspiel zwischen Produktion und Rezeption entstehen. Im folgenden Kapitel hebt die Autorin die Bedeutung von Narrativität im Journalismus hervor und verweist auf das Storytelling als journalistischem Kommunikationsmodus. Der Behauptung, dem Konzept der Narrativität käme in der Journalistik allenfalls eine randständige Existenz zu, mag sich der Rezensent angesichts der breiten angelsächsischen Tradition nicht ganz anschließen.

Das sechste Kapitel widmet sich der Unterscheidung von Fakten und Fiktionen, wobei Lünenborg überzeugend nachzeichnet, wie Unschärfen, Überlappungen und Grenzüberschreitungen zwischen Faktischem und Fiktionalem den Journalismus aufgrund seiner historischen Verbundenheit mit der Literatur stets begleitet haben. Zunehmende Entgrenzungserscheinungen von Faktizität und Fiktionalität seien vor allem im aktuellen Boulevardjournalismus festzustellen. Eine Charakterisierung von Journalismus als „allein auf Fakten basierend" würde somit, so die Autorin, nur eine verkürzte und normativ induzierte Strukturbeschreibung liefern. Dieser Auffassung würden zweifellos auch „traditionelle“ Journalismusforscher vorbehaltlos zustimmen.

Analog dazu plädiert Lünenborg im darauf folgenden Kapitel für eine Sicht, wonach sowohl Information als auch Unterhaltung als konstitutiv für den Journalismus gelten. Sie weist zu Recht darauf hin, dass Information und Unterhaltung zwei voneinander unabhängige Kategorien sind - sowohl auf der Seite der Produktion als auch der Rezeption. Ob und inwieweit sich eine Informations- und/oder Unterhaltungsleistung im Prozess der Rezeption letztlich realisiert, hänge auch vom Rezipienten, seinen Nutzungsmotiven und -gewohnheiten sowie der Rezeptionssituation ab.

Insgesamt bietet der Band eine anregende, provokative und inspirierende Lektüre. Journalismus als kultureller Prozess ist ein Diskussionsbeitrag, der die Perspektive und heuristischen Ansprüche der Cultural Studies auf dem Terrain der Journalismusforschung artikuliert. Lünenborgs Buch bietet überdies genug Reibungsfläche, um notwendige weiterführende Debatten anzustoßen. Denn während die Autorin einerseits der traditionellen Journalismusforschung einen ,verengten Journalismusbegriff“ vorhält, bleibt sie auffällig zurückhaltend im Hinblick auf eine alternative Definition von Journalismus. Vielmehr verschiebt sie die Definitionsleistung auf die Seite des Publikums, womit sie die empirische Journalismusforschung vor das - von ihr nicht gelöste - Dilemma der operationalen Bestimmung des Forschungsgegenstandes stellt.

Dennoch wird Journalismus als kultureller Prozess zweifellos und vollkommen berechtigt einen Platz auf den Leselisten der kulturwissenschaftlich orientierten Journalismusforschung finden. Interessant ist das Buch nicht zuletzt auch für jene, die einen Einstieg in die kulturtheoretische und Cultural-Studies-orientierte Journalismusforschung suchen.

Thomas Hanitzsch 